\title{
EFFECTS OF DIFFERENT VEGETATION ON SOIL MICROBIAL BIOMASS CARBON AND NITROGEN IN NEWLY CULTIVATED LAND
}

\author{
YAN Li ${ }^{1,2,3^{*}}$ AND BAOQIANG ZHANG ${ }^{1}$ \\ Institute of Land Engineering and Technology, Shaanxi Provincial Land \\ Engineering Construction Group Co. Ltd., Xi'an 710021, China
}

Keywords: Newly cultivated land, Vegetation, Soil microbial biomass carbon, Soil microbial biomass nitrogen, Soil microbial quotient

\begin{abstract}
Effects of plant growth on the soil quality and microbial biomass carbon and nitrogen of newlyincreased farmland, providing a scientific basis for the improvement of the newly cultivated land quality and crop selection were studied. Results showed that planting Rosa chinensis Jacq (R), Ardisia crenata Sims (A), Salvia miltiorrhiza (L) and Solanum lycopersicum (S) had significant effects on soil nutrients in cultivated land. The average content of microbial biomass carbon and nitrogen in the rhizosphere soil of the four plants were $91.74-138.55$ and $17.24-79.42 \mathrm{mg} / \mathrm{kg}, 164.05-184.00$ and $28.43-62.31 \mathrm{mg} / \mathrm{kg}, 58.26-73.26$ and $27.04-51.48 \mathrm{mg} / \mathrm{kg}, 183.47$ - 282.00 and $24.89-35.94 \mathrm{mg} / \mathrm{kg}$, respectively. The soil microbial biomass carbon presents as $\mathrm{S}>\mathrm{A}>\mathrm{R}>\mathrm{L}$ at the seedling stage, and $\mathrm{S}>\mathrm{R}>\mathrm{L}>\mathrm{A}$ at the flowering stage. Similarly, soil microbial biomass nitrogen presents as $\mathrm{R}>\mathrm{A}>\mathrm{S}>\mathrm{L}$ at seedling stage and $\mathrm{L}>\mathrm{A}>\mathrm{S}>\mathrm{A}$ at flowering stage. Results fully indicate that the biomass carbon and nitrogen of plants with short growth cycles of newly cultivated land have a small change in the biomass carbon and nitrogen. During the growth process of different vegetation, the changes in rhizosphere soil microbial biomass, carbon-nitrogen ratio and microbial entropy were significantly different. The low shrubs showed an increasing trend, root-growing Salvia miltiorrhiza decreased, while Solanum lycopersicum with the shortest growth cycle showed first increasing and then decreasing trend.
\end{abstract}

\section{Introduction}

Cultivated land resources are the basic resources and important guarantee for agricultural development, rural revitalization, and rural modernization. With the advancement of the new urbanization process, the quantity and quality of cultivated land have undergone significant changes (Zhao et al. 2020, Kong 2021, Yuan et al. 2021). Numerous practices have proved that strict implementation of land use control, implementation of cultivated land compensation, and increased investment in high-standard farmland construction in major grain production areas are important means to balance cultivated land protection and regional development, and are also the key to China's resolutely maintaining the red line of 1.8 billion acres of cultivated land (Shao et al. 2019, Zhou et al. 2020).

Soil is the important habitat for microorganisms to inhabit and multiply. Microbial biomass mainly includes bacteria, fungi, protozoa and other organisms whose body volume is less than $5 \times 10^{3} \mu^{3}$ in inactivated plants in the soil, which characterizes the quality of soil, the intensity of material metabolism, and even the quality of land improvement and cultivated land being feature indicators for upgrading (Guo 2014, Pan et al. 2019). The metabolism of soil microorganisms

*Author for correspondence: <liyan_hhu@163.com>. ${ }^{1}$ Shaanxi Provincial Land Engineering Construction Group Co. Ltd., Xi'an-710075, China. ${ }^{2}$ Key Laboratory of Degraded and Unused Land Consolidation Engineering, the Ministry of Natural Resources, Xi'an-710021, China. ${ }^{3}$ Shaanxi Provincial Land Consolidation Engineering Technology Research Center, Xi'an-710021, China. 
affects the cycle of nutrient elements such as carbon and nitrogen. Correspondingly, the carbon and nitrogen content of microbial biomass significantly affects the metabolism of the microbial community, which in turn affects the physical and chemical properties and nutrient content of the soil, and to a certain extent reflects the soil quality and the suitability of plant growth (Tang et al. 2016, Li et al. 2019). Zhao et al. (2013) reported that four typical vegetations in the loess hilly area have significant effects on soil microbial biomass carbon, nitrogen, and phosphorus, and have significant correlations with soil water content and total nitrogen. Vegetation types have a close relationship with soil microbial biomass. Its physical and chemical properties have a significant impact. Quan (2020) studied the effects of exogenous fertilization on corn and soil microbial biomass and showed that soil carbon and nitrogen, microbial biomass, and plant growth and development have significant effects on soil quality. Plant rhizosphere soil microorganisms are closely related to changes in soil quality and fertility (Liu et al. 2018, Feng et al. 2021). In view of the low fertility level, poor physical and chemical properties, and poor soil quality of newly added cultivated land, the present study was conducted the remediation of inferior forest land in the Loess Plateau as a starting point, combined with regional ecological restoration and the requirements of natural environment and economic development, and evaluated the quality of newly added cultivated land. Research on the promotion and cultivation of suitable crops were carried out by -Wang et al. (2019) and Zhang et al. (2020).

Based on the premise of protecting the ecological environment and improving soil quality, four cash crops with different growth cycles were selected for pilot trials of soil improvement of newly added cultivated land. Effects of different vegetation on the soil nutrients and microorganisms of newly cultivated land were also explored for regional land improvement and increase. The improvement of cultivated land quality and the construction of high-standard farmland would provide scientific basis.

\section{Materials and Methods}

According to the remediation of inferior forest land in the Loess Plateau, pilot trials of cultivated land utilization and quality improvement have been carried out. The experimental soil is yellow loamy soil, and the experimental plants are four typical economic crops, including Rosa chinensis jacq (R), Salvia miltiorrhiza (L), Ardisia crenata Sims (A) and Solanum lycopersicum (S). Before planting, the tillage layer $(0-30 \mathrm{~cm})$ was tilled evenly, and the depth of each test field was kept the same. At the same time, nitrogen fertilizer (46.7\%) $32 \mathrm{~g} / \mathrm{m}^{2}, \mathrm{P}_{2} \mathrm{O}_{5}(48 \%) 31 \mathrm{~g} / \mathrm{m}^{2}$ and $\mathrm{K}_{2} \mathrm{O}(50 \sim 52 \%) 30 \mathrm{~g} / \mathrm{m}^{2}$ and biological nutrient-based fertilizer $1000 \mathrm{~g} / \mathrm{m}^{2}$, respectively were applied to ensure the necessary nutrient requirements for plant growth (Table 1).

Table 1. Different vegetation treatments and fertilization situation.

\begin{tabular}{llccc}
\hline \multirow{2}{*}{ Plant } & Fertilizer & \multicolumn{3}{c}{ Total fertilization $\left(\mathrm{g} / \mathrm{m}^{2}\right)$} \\
\cline { 3 - 5 } & $\mathrm{N}$ & Ground fertilizer & Additional fertilizer & Total fertilization \\
\hline \multirow{3}{*}{$\mathrm{R}, \mathrm{S}, \mathrm{L}, \mathrm{A}$} & $\mathrm{P}_{2} \mathrm{O}_{5}$ & 31 & 0 & 32 \\
& $\mathrm{~K}_{2} \mathrm{O}$ & 30 & 0 & 31 \\
& biological nutrient & 1000 & 0 & 30 \\
& base fertilizer & & 1000 & 2000 \\
\hline
\end{tabular}


According to the growth cycle characteristics of different plants, three different $(40 \mathrm{~cm} \times 40$ $\mathrm{cm}$ ) plants were randomly selected during the critical period of vegetation growth from March to November 2020 to collect the rhizosphere soil of each type of vegetation. In particular, the Solanum lycopersicum has a short growth cycle, and the rhizosphere soil was collected during the seedling, flowering and fruiting stages. Vegetation rhizosphere soil samples were collected using the "root shaking method". After the entire root system of the plant was excavated, the surrounding soil was stripped, and the soil attached to the root surface $(0-4 \mathrm{~mm})$ was collected (Chen et al. 2021). Three parallel samples of rhizosphere soil were collected each time, and each sample was divided into two groups: a group of soil samples were put into sample bags and stored at $4{ }^{\circ} \mathrm{C}$, and chloroform fumigation-extraction method was used to determine the soil microbial biomass carbon and nitrogen content. Another set of soil samples were air-dried and sieved to determine the total nitrogen and organic matter content and total organic carbon of the soil, using the methods of NYT53-1987, NY/T1121.6-2006 and HJ695-2014. The calculation formula for the analysis of the results of this research is as follows:

Soil microbial biomass carbon nitrogen ratio $(\mathrm{SMBC} / \mathrm{SMBN})=$ soil microbial biomass carbon/soil microbial biomass nitrogen.

Soil microbial quotient $(\mathrm{SMQ})=$ soil microbial biomass carbon $/$ total soil organic carbon $\times$ $100 \%$.

\section{Results and Discussion}

During the growth process of different types of vegetation, the total nitrogen and organic matter content of the rhizosphere soil showed obvious differences (Wan et al. 2021). Figure 1 showed that during the seedling-flowering-fruiting (SS-BP-FR) period of Solanum lycopersicum (S), the concentration of total nitrogen and organic matter in the rhizosphere soil dropped remarkably from $1.21 \mathrm{~g} / \mathrm{kg}$ and 16.30 to 0.45 and $5.74 \mathrm{~g} / \mathrm{kg}$, respectively. Similarly, the concentration of total nitrogen and organic matter in the rhizosphere soil of Ardisia crenata Sims (A) at the seedling stage was slightly higher than that at the flowering stage. Conversely, the total nitrogen and organic matter concentrations in the rhizosphere soil during the growth of Rosa chinensis Jacq (R) and Salvia miltiorrhiza (L) showed the following law: BP > SS, total nitrogen increased from 0.92 and $0.76 \mathrm{~g} / \mathrm{kg}$, respectively 1.20 and $0.91 \mathrm{~g} / \mathrm{kg}$, an increase of 30.59 and $19.15 \%$. Correspondingly, organic matter increased by 5.30 and $4.79 \mathrm{~g} / \mathrm{kg}$. On the whole, the content of total nitrogen and organic matter in the rhizosphere soil during the growth of different vegetations showed the following rules: $\mathrm{S}>\mathrm{A}>\mathrm{R}>\mathrm{L}$ at the seedling stage, $\mathrm{R}>\mathrm{A}>\mathrm{L}>\mathrm{S}$ at the flowering stage. That is, the concentration of total nitrogen and organic matter in $\mathrm{S}$ rhizosphere soil with the shortest growth cycle decreased the most (Zhou 2019).
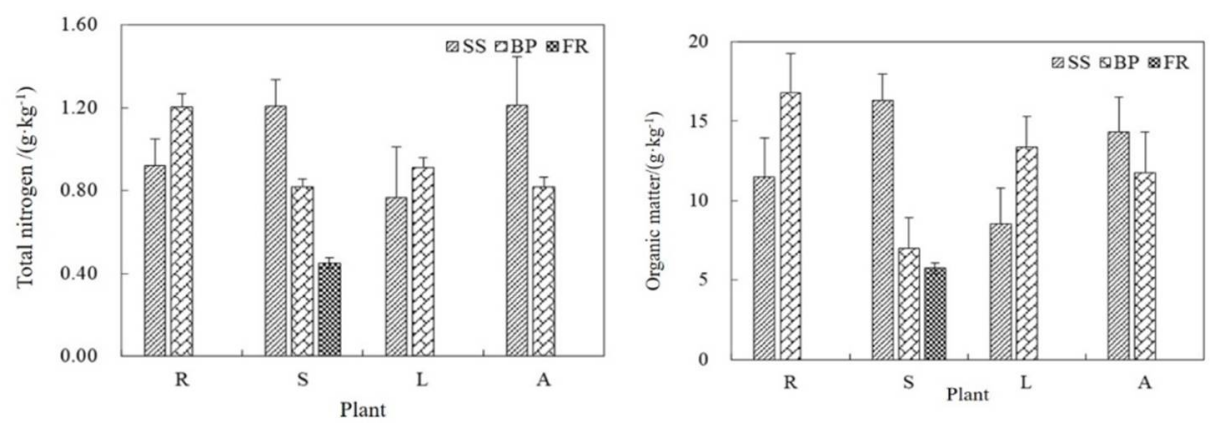

Fig. 1. Effects of different types of vegetation on soil nutrients in newly cultivated land. 
The general tendency of SMBC changes in rhizosphere soil was consistent during the seedling and flowering stages of different types of vegetation (Zhu 2018). It is apparent from Fig. 2 that the SMBC of the rhizosphere soil of R and A showed a downward trend from the seedling stage to the flowering stage, and the range of SMBC content was $91.74-138.55$ and $164.05-184.00 \mathrm{mg} / \mathrm{kg}$. From the seedling stage to the flowering stage to the fruiting stage, SMBC in the rhizosphere decreased continuously, and its content varied from $183.47-282.00 \mathrm{mg} / \mathrm{kg}$. The difference is that the SMBC content of L rhizosphere soil increased from 58.26 to $73.26 \mathrm{mg} / \mathrm{kg}$, with an increase of $25.75 \%$. In summary, the change of SMBC content in rhizosphere soil during the growth of different vegetation showed the following laws: $\mathrm{S}>\mathrm{A}>\mathrm{R}>\mathrm{L}$ at seedling stage, $\mathrm{S}>\mathrm{R}>\mathrm{L}>\mathrm{A}$ at flowering stage, which means that the content of SMBC in the rhizosphere soil of Solanum lycopersicum with the shortest growth cycle is relatively the highest and the smallest drop. On the other hand, the SMBN content in the rhizosphere changes greatly during the growth of vegetation. The SMBN in the rhizosphere soil of S decreased first and then increased from the seedling stage to the flowering stage to the fruiting stage, and the range of SMBN content was $24.89-35.94$ $\mathrm{mg} / \mathrm{kg}$. The SMBN of R and A rhizosphere soils was found to reduce significantly, and the SMBN content ranged from $17.24-79.94$ and $28.43-62.31 \mathrm{mg} / \mathrm{kg}$, respectively. On the contrary, the SMBN content of the L rhizosphere soil increased from 27.04 to $51.48 \mathrm{mg} / \mathrm{kg}$. The variation of SMBN content in rhizosphere soil during the growth of the four vegetations was: $\mathrm{R}>\mathrm{A}>\mathrm{S}>\mathrm{L}$ at the seedling stage and $\mathrm{L}>\mathrm{A}>\mathrm{S}>\mathrm{A}$ at the flowering stage. The SMBN content in the rhizosphere of $\mathrm{R}$ has the largest decrease.
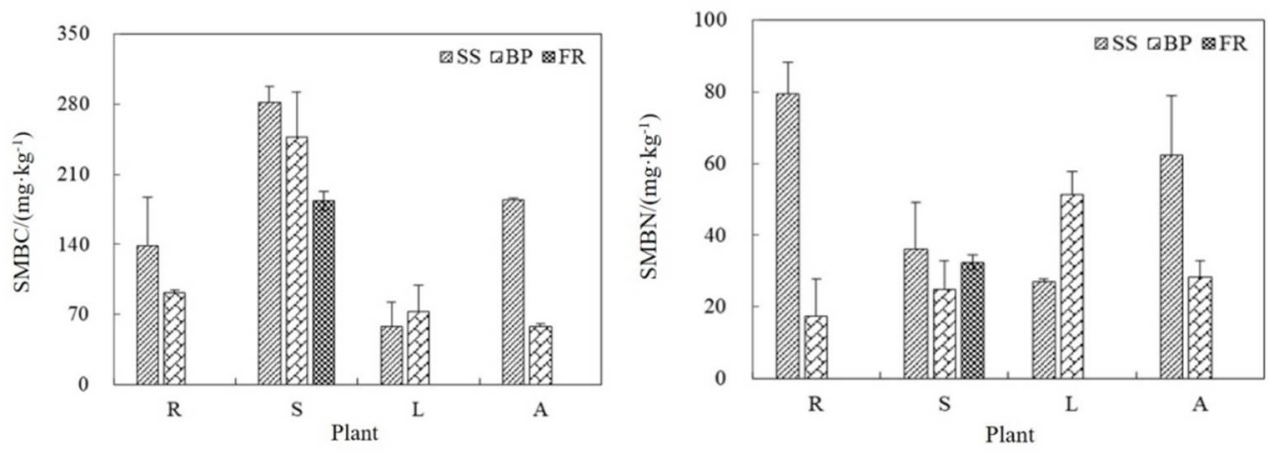

Fig. 2. Changes of SMBC and SMBN in rhizosphere soil of newly cultivated land vegetation.

Furthermore, the SMBC/SMBN of the rhizosphere soil during the growth of different vegetations were not the same (Fig. 3). The SMBC/SMBN at the flowering stage of $\mathrm{R}$ and A was significantly higher than that at seedling stage (BP > SS). In the same way, the SMBC/SMBN of S at different growth periods increased first and then decreased (BP > SS > FR), and the ratio ranged from 5.66 to 9.91. Different from the other three types of vegetation, the L seedling stage SMBC/SMBN was 2.15, which was 1.42 higher than the flowering stage (BP < SS). In addition, the variation of soil microbial entropy (SMQ) in each growth period of the four vegetations was relatively small. The SMQ of rhizosphere soil from L seedling stage to flowering stage decreased from 2.08 to 0.94 (a decrease of 55\%). The SMQ of A increased slightly from the seedling stage (2.21) to the flowering stage. Solanum lycopersicum SMQ increased from 2.98 to 6.10 at the seedling stage (flowering stage), and then decreased to 5.51 as the Solanum lycopersicum matured, showing a trend of increasing first and then decreasing. However, SMQ decreased slightly during the L-long process, and the SMQ value decreased from 1.17 to 0.95 . 

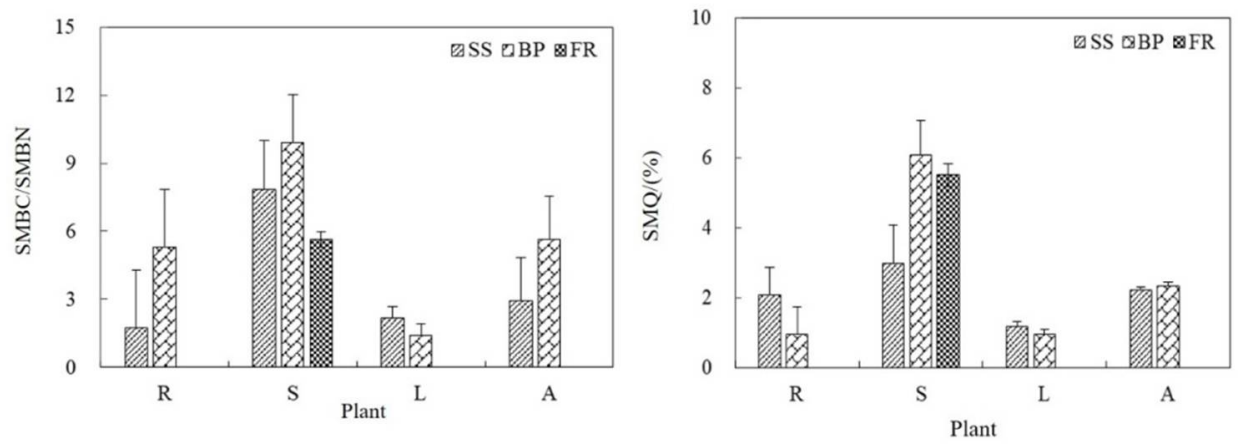

Fig. 3. Changes of SMBC/SMBN and SMQ in rhizosphere soils of different types of vegetation.

Significance analysis showed that rhizosphere soil SMBC of different types of vegetation was extremely significantly correlated with total nitrogen, and significantly positively correlated with SMBN and organic carbon (Table 2). The soil microbial biomass nitrogen was extremely significantly positively correlated with total nitrogen and soil moisture content, and significantly positively correlated with organic carbon. The soil moisture content was significantly correlated with microbial biomass carbon, total nitrogen, and organic carbon, which indicates that the plant rhizosphere soil microbial biomass is closely related to soil organic carbon, total nitrogen, and moisture content in the process of newly cultivated land improvement and quality improvement.

Table 2. The correlation between soil microbial biomass carbon and nitrogen and soil properties in different vegetation rhizospheres.

\begin{tabular}{lccccc}
\hline Correlation coefficient & SMBC & SMBN & Total nitrogen & Organic carbon & Soil moisture content \\
\hline SMBC & 1 & $0.838^{*}$ & $0.989^{* *}$ & $0.979^{*}$ & 0.122 \\
SMBN & & 1 & $0.966^{* *}$ & $0.820^{*}$ & $0.995^{* *}$ \\
Total nitrogen & & & 1 & $0.969^{*}$ & 0.262 \\
Organic carbon & & & & 1 & 0.211 \\
Soil moisture content & & & & & 1 \\
\hline
\end{tabular}

*means there is a significant correlation $(\mathrm{p}<0.05)$, and **means there is a very significant correlation $(\mathrm{p}<0.01)$.

Plant growth and development occur under the combined effects of its shape, nutritional status, and environmental conditions (Xiao et al. 2021). As the growth period advances, S continuously absorbed soil nutrients from seedling stage to flowering stage and fruiting stage. The total nitrogen and organic matter content of the rhizosphere soil continued to decrease. During the growth and development of Solanum lycopersicum roots, SMBC decreased, and SMBN first decreased and then increased. Correspondingly, SMBC/SMBN showed a rule that the seedling stage increased to the highest value at the flowering stage, and then decreased with the fruiting stage.

The main growth parts of evergreen low shrubs $\mathrm{R}$ and $\mathrm{A}$ are the stems and leaves on the ground, and their root system is relatively slow (Huang et al. 2015, Li et al. 2020). At the same time, affected by factors such as soil moisture and soil plowing, soil nutrients, SMBC and SMBN decreased to varying degrees during the rapid growth of the above-ground part from the seedling 
stage to the flowering stage, while SMBC/SMBN showed an increasing trend. On the other hand, the perennial herb L is mainly based on root growth. During the period from the seedling stage to the end of the flowering period, its roots continuously absorb soil nutrients and increase rapidly (Chen et al. 2019). At the same time, secretions and sloughs also provide sufficient nitrogen and carbon sources for microorganisms, which promote the increase of SMBC and SMBN in rhizosphere soil, and SMBN is higher (Zhang et al. 2020, Guo et al. 2021).

Soil microbial entropy characterizes soil quality and its carbon dynamics, directly reflecting the proportion of soil active organic carbon. During the growth period of different plants, the dynamics of microbial entropy in the rhizosphere soil vary significantly. The microbial entropy of the rhizosphere soil in the seedling-flowering-fruiting stage of $\mathrm{S}$ increased first and then decreased. The soil microbial entropy of the rhizosphere soil during the seedling-floweringfruiting stage of $\mathrm{S}$ increased first and then decreased. This might be due to the rapid growth of $\mathrm{S}$ seedling and flowering stage, and the large consumption of rhizosphere microbe growth and metabolism increased SMBC/SMBN. However, due to the low soil fertility of the newly-increased farmland, factors such as insufficient soil nutrient supply, low water content and the gradual decrease of plant physiological activities as plants grow, which affect the soil microbial activities, and ultimately lead to the reduction of SMBC and SMBN, and the continuous soil microbial entropy reduce ( $\mathrm{Li}$ et al. 2020). The study showed that low shrubs $\mathrm{R}$ and $\mathrm{A}$ required different nutrient content during the growth process, soil SMBC and total organic carbon change range were different, and the corresponding soil bio-entropy change trends were also significantly different. The soil microbial entropy of planting $\mathrm{R}$ is more significant. Decreased, but the soil microbial entropy of planting A increased slightly. This might be related to factors such as plant growth rate, rhizosphere characteristics and nutrient absorption capacity during key growth periods.

Different types of vegetation have a significant impact on the soil quality of newly- increased farmland. Experiments have shown that plant growth directly affected the content of soil organic matter and total nitrogen. Moreover, the effect of $\mathrm{S}$ with a short growth cycle on soil nutrients was significantly higher than that of perennial evergreen plants with a long growth cycle (such as R, L and A). The differences in SMBC/SMBN and microbial entropy of rhizosphere soil during the growth of different vegetations were obvious. The SMBC/SMBN of the rhizosphere soil of low shrubs ( $\mathrm{R}$ and $\mathrm{A}$ ), which mainly grew above ground increased significantly, while the SMBC/SMBN and microbial entropy of L rhizosphere soil which mainly grew underground rhizomes decreased slightly. In addition, $\mathrm{S}$ has the shortest growth cycle, which showed a trend of first increase and then decrease in the rhizosphere soil SMBC/SMBN and microbial entropy during its critical growth period (seedling stage-flowering stage-fruiting stage). In summary, the quality of cultivated land with low soil nutrient content and quality needs to be improved, and vegetation with a short growth cycle can quickly change its quality. However, the other agrotechnical measures need to be assisted to effectively improve soil quality and upgrade prefecture level.

\section{Acknowledgements}

The authors are grateful to the Technology Innovation Center for Land Engineering and Human Settlements, Shaanxi Land Engineering Construction Group Co. Ltd. and Xi'an Jiaotong University (2021WHZ0094), and the Shaanxi Provincial Land Engineering Construction Group internal research project (DJNY2021-24) for financial support to the present study. 


\section{References}

Chen XY, He C, Yan BB, Li WB, Gen YY, Xia GH, Hou JL, Wang WQ, Song Y and Zhu GM 2019. Effect of fertilization combination of nitrogen, phosphorus, and potassium on yield and quality of Salvia miltiorrhiza. Chinese Tradition. Herbal Drugs 50(3): 722-730.

Chen XY, Yao XD, Zeng WJ and Wang W 2021. Spatial pattern and driving factors of soil microbial biomass carbon in grassland in Northern Agro-Pastoral Transition Zone. Acta Scientiarum Naturalium Univ. Pekinensis 57(02): 250-260.

Feng HL, He HH, Xu Q, Li XL, Zhang F, Ji XM, Xu CS, Ren TB and Liu GS 2021. Effects of combined application of biochar and nitrogen fertilizer on soil microorganism and carbon and nitrogen content in tobacco growing soil. Soil Fertilizer Sci. China. doi:10.11838/sfsc.1673-6257.20414.

Guo HB 2014. Effects of tillage and crop residue management soil biological properties and plant growth in a winter wheatear-summer maize cropping system. Henan Agricultural Univ. Master's thesis. pp.17-37.

Guo M, Huang Y, Li HM, Zhang HR, Zhou Y, Gao ZM and Ding MY 2021. Effects of salinity, alkalinity, temperature, and their interactions on seed germination and seedling growth of Salvia miltiorrhiza. Pratacultural Sci. 38(4): 664-672.

Huang SH, Qiu FY and Hong YP 2015. Study on seed germination of ornamental plant Ardisia crenata. J. Longyan Univ. 33(2): 94-99.

Kong XB 2021. Transition objectives, contents and ways of cultivated land protection in China under the background of rehabilitation system. J. Soc. Sci. Hunan Normal Univ. 50(3): 1-12.

Li CY, Hao YH, Xue YL, Wang Y and Dang YH 2020. Effects of long-term fertilization on soil microbial biomass carbon, nitrogen, and phosphorus in the farmland of the Loess Plateau, China. J. Agro-Environ. Sci. 39(8): 1783-1791.

Li CY, Zhang EH and Ding ML 2020. Effects of plant growth regulators on the survival of rose cuttings. Jiangxi Agriculture 10: 95+99.

Li P, Tuerhanbai M, Tian D and Feng ZZ 2019. Seasonal dynamics of soil microbial biomass carbon, nitrogen and phosphorus stoichiometry across global forest ecosystems. Chinese J. Plant Ecol. 43(06):532-542.

Liu JH, Xu H, Wang Y, Li H and Shen WD 2018. Evaluation of ecological risk and carbon fixation from land use change: A case study of Huanghua City, Hebei Province. Chinese Journal of Eco-Agriculture 26(8): 1217-1226.

Pan XC, Tang HM, Xiao XP, Li C, Tang WG, Cheng KK, Guo LJ, Huang GL and Wang K 2019. Effects of different soil tillage and returning crop residues systems on soil microbial biomass carbon and nitrogen under Chinese milk vetch and double-cropping rice field. Ecol. Environ. Sci. 28(8): 1585-1595.

Quan JZ 2020. Effects of different fertilization levels and corn planting on soil microbial biomass carbon and nitrogen content. Grain Sci. Technol. Econ. 45(09): 105-106.

Shao YJ and Yuan XF 2019. Zoning of cultivated land quality improvement potential based on limiting factor analysis. Chinese Journal of Ecology 38(8): 2442-2449.

Tang XL, WEI JB, Zhou LH, Xu HQ, Zou YB and Tang JW 2016. The dynamic effect of different tillage methods on soil microbial carbon and nitrogen in rice paddy. Crop Research 30(3): 282-287.

Wan XF, Wang S, Zhang Y, Wang TL, Ge Y, Gao SX, Kang CZ, Lü CG, Wang RS and Guo LP 2021. Effect of combined application of inorganic and organic fertilizers on growth and quality of Salvia miltiorrhiza. China J. Chinese Materia Medica 46(08): 1927-1934.

Wang LY, Anna H, Zhang LY, Xiao Y, Wang YQ, Xiao Y, Liu JG and Ouyang ZY 2019. Spatial and temporal changes of arable land driven by urbanization and ecological restoration in China. Chinese Geogr. Sci. 29(5): 809-819.

Xiao Y, Huang ZG, Xiao HX, Li YF and Peng WX 2021. Changes of soil microbial biomass carbon, nitrogen and enzyme activities in East Dongting Lake Wetlands at different water levels. Chinese J. Appl. Ecol. 32(08): 2958-2966. 
Yuan CC, Zhang DX, Liu LM and Ye JW 2021. Regional characteristics and spatial-temporal distribution of cultivated land change in China during 2009-2018. Trans. Chinese Soc. Agricul. Engineer. 37(1): 267-278.

Zhang K 2020. Establish an efficiency propagation system and study the affection on the growth and secondary metabolites of salvia miltiorrhiza micro-shoots by rare earth elements. Inner Mongolia Agricultural Univ. master's thesis. pp. 28-33.

Zhang YX, Wang YK and Fu B, et al 2020. Changes in cultivated land patterns and driving forces in the Three Gorges Reservoir area, China, from 1992 to 2015. J. Mountain Sci. 17(1): 203-215.

Zhao T, Yan H, Jiang YL, Huang YM and An SS 2013. Effects of vegetation types on soil microbial biomass C, N, P on the Loess Hilly Area. Acta Ecol. Sinica 33(18): 5615-5622.

Zhao YH and Tan YZ 2020. Spatial-temporal changes of cultivated land use at provincial level since second national land survey in China. Bull. Soil Water Conserv. 40(1): 204-212.

Zhou EL 2019. Effects of different forms of nitrogen on the growth and nutrient absorption of Salvia miltiorrhiza at seedling stage. J. Chinese Med. Materials 42(02): 260-263.

Zhou XH 2020. Quality Grades of newly cultivated land and factors influencing grain productivity in loess tableland area. Bull. Soil Water Conserv. 40(4): 237-243.

Zhu LX 2018.Effects of different management practices on soil carbon and nitrogen and related microbial processes in rain-fed farmlands. Northwest A\&F Univ. doctoral dissertation. pp. 55-75.

(Manuscript received on 22 June, 2021; revised on 27 September, 2021) 